\title{
The era of uniportal video-assisted thoracoscopic surgery
}

\author{
Monica Pastina ${ }^{1}$, Cecilia Menna $^{2}$, Claudio Andreetti ${ }^{2}$, Mohsen Ibrahim $^{2}$ \\ ${ }^{1}$ Division of Thoracic Surgery, Policlinico "A. Gemelli” Hospital, Catholic University of Sacred Heart, Rome, Italy; ${ }^{2}$ Division of Thoracic Surgery, \\ Sant'Andrea Hospital, University of Rome "Sapienza", Rome, Italy \\ Correspondence to: Mohsen Ibrahim. Division of Thoracic Surgery, Sant'Andrea Hospital, University of Rome "Sapienza”, Via di Grottarossa 1035, \\ 00189, Rome, Italy. Email: mohsen.ibrahim@uniroma1.it. \\ Provenance: This is an invited Editorial commissioned by the Section Editor Jianfei Shen (Taizhou Hospital of Zhejiang Province, Wenzhou Medical \\ University, Taizhou, China). \\ Comment on: Shen Y, Wang H, Feng M, et al. Single- versus multiple-port thoracoscopic lobectomy for lung cancer: a propensity-matched study. Eur \\ J Cardiothorac Surg 2016;49 Suppl 1:i48-53.
}

Submitted Jan 23, 2017. Accepted for publication Feb 15, 2017.

doi: $10.21037 /$ jtd.2017.02.97

View this article at: http://dx.doi.org/10.21037/jtd.2017.02.97

The first anatomic lung resection was performed through video-assisted thoracoscopic surgery (VATS) in 1992. Since then, VATS technique has been evolving in terms of clinical indications and surgical technique for early stage (I or II) non-small cell lung cancer (NSCLC) treatment.

Accordingly, thoracotomy has been replaced by multiportal-VATS (M-VATS) (4-3-2 surgical ports) moving towards uniportal-VATS (U-VATS) (single surgical port). Reduced number and size of surgical incisions led to a decreased inflammatory response less, postoperative pain and better cosmetic results for patients (1).

The transition from the thoracotomy to VATS determined a reduction in postoperative complication, chest tube permanence, postoperative length of stay and postoperative pain resulting in lower costs for VATS procedures $(2,3)$.

Early on U-VATS was used for wedge resection, treatment of primary spontaneous pneumothoraxes, sympathectomy, pericardial window, removal of small mediastinal masses and evacuation of hemothorax (4).

In the last ten years, Gonzalez-Rivas et al. developed this technique for even more complex resections like lobectomy and sleeve segmentectomy, lobectomy, pneumonectomy, chest wall resection and pulmonary artery and bronchial reconstruction (sleeve lobectomy) (5).

In literature, there are a number of studies comparing U-VATS versus M-VATS, but they are all retrospective studies.

The introduction of new technologies such as HD and 3D lens camera, flexible thoracoscopy, extremely fine needlescopic, thinner and ergonomic surgical instruments, smaller and angled mechanical stapler has been promoting the development of U-VATS. Thus, the use of the new generation flexible $5 \mathrm{~mm} 0^{\circ}$ videoscope with an 85 -degree rotating tip may allow a better view of posterior hilum thus facilitating the dissection and lymphadenectomy (6).

Moreover, in UVATS the introduction of instruments is parallel to videoscope obtaining a similar point of view to that of open surgery unlike in M-VATS approach where a triangular geometric configuration consents a maximal convergence of surgical instruments but often with an interference with optical source $(7,8)$. For these reasons U-VATS is not considered as a natural evolution of M-VATS by many authors, but rather a thoracotomy development.

For the U-VATS lobectomy the surgical incision (3$4 \mathrm{~cm}$ ) is performed at the fourth intercostal space along the anterior axillary line for upper lobe resection and at the fifth intercostal space for middle and inferior lobe resection. In the latter case, the dissection order is generally the vein first, then the artery and the bronchus, while for the upper lobe is better to start with the dissection of the artery (9). Furthermore, in UVATS trocars or rib retractors are not used reducing the insult to the intercostal nerves.

The use of soft tissue retractors (like Alexis ${ }^{\circledR}$ ) to facilitate the introduction of instruments may be suggested. Anterior approach is generally preferred.

The surgeon is located in front of the patient and the assistant can be located either in front or alongside of the 
surgeon $(6,10)$. At the end of surgery, the chest tube is inserted at the posterior extremity of the incision.

The lymphadenectomy is essential in lung cancer surgery for staging and tumor local control. The effectiveness of lymphadenectomy whether in VATS or thoracotomy still is controversial. Many authors showed that, mostly using proper tools, same lymphadenectomy results can be obtained in VATS and thoracotomy approach, and better results have been showed in U-VATS than in M-VATS (11).

Liu et al. showed that both in U-VATS and in M-VATS the quantity and quality of dissected lymph nodes are the same. Nevertheless, the exposure of the left-side subcarinal area in U-VATS is sometime could be quite challenging, comparing to M-VATS (12).

Mu et al., in a study published in 2015 showed a less number of dissected lymph nodes $(7.02 \pm 8.60 v s .13 .34 \pm 9.26$, $\mathrm{P}<0.001)$ and a less number of lymph node stations examined $(4 \pm 1$ vs. $6 \pm 1, \mathrm{P}<0.001)$ in $\mathrm{U}$-VATS than those obtained in M-VATS lobectomy (13).

Moreover it is still strongly debated the issue of surgical time. Tamura et al. (14) highlighted in their experience the longer operative time for bullectomy, partial lung resection and thymectomy in U-VATS, same as Lin et al. for U-VATS lobectomy (U-VATS $132.3 \pm 13.2$ vs. M-VATS $105.4 \pm 12.5$, $\mathrm{P}<0.05)(15)$.

Instead, Shen et al. in their study showed that the duration of lobectomy in U-VATS was shorter than in M-VATS $(65.7 \pm 14.8$ vs. M-VATS $81.3 \pm 13.6, \mathrm{P}<0.001)$, although the duration of lymphadenectomy was longer in U-VATS that in M-VATS (9).

In 2011 Gonzalez et al. described the initial experience in VATS lobectomy, comparing three different periods of time (first period from 2007 to 2008, second period from 2008 to 2009 and third period from 2009 to 2010) and showing that the learning curve is crucial for the improvement of the operative time in VATS lobectomy; consequently, the third period is characterized by lower operative times than the previous ones (16).

The transition from thoracotomy to the U-VATS may require several steps. The surgeon could have less difficulty to switch from the traditional VATS to U-VATS, having already gained more dexterity with instruments and methods (17).

Many authors have directed their work toward the difference in postoperative outcomes between U-VATS, M-VATS and thoracotomy. Numerous studies showed that VATS is associated to a lower morbidity ( $29.1 \%$ vs. $31.7 \%)$ and mortality ( $1 \% v s .1 .9 \%)$ comparing to thoracotomy.
VATS is associated with lower major cardiopulmonary complications, atrial fibrillation, atelectasis and wound infection. Moreover, VATS is characterized by a shorter length of stay. For these reasons, VATS is considered the best approach especially for elderly patient (over 70 years old) and for patients with FEV1 <40\% (18).

However, the most important goal has been achieved by the decrease of postoperative pain due to a single incision without rib spreading. This issue led to a reduction of hospitalization and to a rapid return to work (19) resulting in lower cost and reduced time to start adjuvant chemotherapies. Nevertheless, the oncological benefits and 5-year survival appear equivalent in VATS compared with open surgery for stage I non-small-cell lung cancer (Regional lymphadenectomy, nodal upstaging, overall and disease-free survival were similar between the two techniques) (20).

Moreover, although the benefits of VATS compared to thoracotomy are now well known, there are conflicting opinions regarding the difference in postoperative outcomes between U-VATS and M-VATS.

Harris et al. (21) in their review including eight large retrospective studies showed that U-VATS, compared with M-VATS, was associated to a shorter duration of chest tube permanence and lower morbidity, without significant differences in number of lymph nodes dissected, conversion rate to open thoracotomy or operative time. Furthermore, Dai and colleagues in their propensitymatched comparative analysis showed a reduction of intraoperative blood loss and postoperative pain with a higher patient satisfaction score in U-VATS (22). French et al. did not find significant differences in conversion rate, perioperative bleeding, mortality and median operative time between the two techniques (23). Chung et al. in their retrospective experience did not find differences in operative time, chest tube permanence, postoperative 30-day mortality, number of removed lymph nodes, reoperation rate and length of postoperative hospital stay between the two groups (M-VATS lobectomy vs. U-VATS lobectomy) (24).

Moreover, a large number of intraoperative complications, such as intraoperative bleeding, can be controlled both in conventional VATS than in U-VATS with a lower conversion rate to thoracotomy (25), reaching operative time similar to open surgery. This is due to the increasing experience of the surgeons and the development of surgical techniques and surgical instruments shaped especially for the U-VATS procedure, with the recent 
advent of high definition camera.

In the present study, Shen et al. compared patients with NSCLC underwent thoracoscopic lobectomy through M-VATS or U-VATS by using the propensity-matched analysis, presenting detailed tables and well describing the surgical technique.

This paper is interesting because the authors enrolled a large number of patients (411 patients between 2013 and 2014). The two samples are extremely homogeneous. The study is the first report present in literature where the propensity-matched analysis is used to compare the uniportal technique with the multiportal one (there are studies where this statistical test is used to compare the open technique versus VATS). More interestingly, the study showed the comparison between data analyzed with propensity score-matched test and data analyzed not with this test, confirming how the propensity provided stronger homogeneous results.

Finally, the first 15 cases of U-VATS lobectomy were eliminated from the study to limit the effect given by the learning curve. Thus, the study is statistically valid.

Another strong point of this paper is that all surgical procedures, despite the high number of patients enrolled, were performed by the same surgeon. More in detail, statistically significant results were obtained for the operative time $(\mathrm{P}>0.001)$. In the U-VATS group, the operative time was shorter than the M-VATS group $(65.7 \pm 14.8$ vs. $81.3 \pm 13.6)$, while the operative time for the lymphadenectomy was longer $(29.6 \pm 16.7$ vs. $17.4 \pm 13.3)$.

By contrast, this is a retrospective study. Moreover, the sample size is small for the propensity-matched method. Given that, the propensity score method is generally used on large samples, often recruited from international databases.

In conclusion, U-VATS is proved to be a viable alternative to open surgery, although advantages over traditional VATS especially from the point of view of outcomes are not clear yet. Indeed, there are not strong differences between the two techniques. However, it has been proved that the U-VATS is a technique with a favorable learning curve.

Many retrospective studies have already confirmed the advantage of single port technique compared with multiport in term of better postoperative pain control, easier surgical approach and better cosmetic results in thoracic surgery.

Future prospective studies and longer follow-up may reveal new benefits and novel indications for U-VATS procedure.

\section{Acknowledgements}

None.

\section{Footnote}

Conflicts of Interest: The authors have no conflicts of interest to declare.

\section{References}

1. Vanni C, Menna C, Andreetti C, et al. Transition from multiple-port to single-port video-assisted thoracic surgery pulmonary lobectomy: a technical evolution? Video-assist Thorac Surg 2016;1:29.

2. Andreetti C, Menna C, Ibrahim M, et al. Postoperative pain control: videothoracoscopic versus conservative mini-thoracotomic approach. Eur J Cardiothorac Surg 2014;46:907-12.

3. Paul S, Altorki NK, Sheng S, et al. Thoracoscopic lobectomy is associated with lower morbidity than open lobectomy: a propensity-matched analysis from the STS database. J Thorac Cardiovasc Surg 2010;139:366-78.

4. Reinersman JM, Passera E, Rocco G. Overview of uniportal video-assisted thoracic surgery (VATS): past and present. Ann Cardiothorac Surg 2016;5:112-7.

5. Gonzalez-Rivas D, Yang Y, Stupnik T, et al. Uniportal video-assisted thoracoscopic bronchovascular, tracheal and carinal sleeve resections. Eur J Cardiothorac Surg 2016;49 Suppl 1:16-16.

6. Ibrahim M, Menna C, Andreetti C, et al. Flexible videoscope for thoracoscopic lobectomy: evolution of uniportal technique. Surg Endosc 2015;29:2056-9.

7. Bertolaccini L, Rocco G, Viti A, et al. Geometrical characteristics of uniportal VATS. J Thorac Dis 2013;5 Suppl 3:S214-6.

8. Chang JM, Kam KH, Yen YT, et al. From biportal to uniportal video-assisted thoracoscopic anatomical lung resection: A single-institute experience. Medicine (Baltimore) 2016;95:e5097.

9. Shen Y, Wang H, Feng M, et al. Single- versus multipleport thoracoscopic lobectomy for lung cancer: a propensity-matched study. Eur J Cardiothorac Surg. 2016;49 Suppl 1:i48-53.

10. Hernandez-Arenas LA, Lin L, Yang Y, et al. Initial experience in uniportal subxiphoid video-assisted thoracoscopic surgery for major lung resections. Eur J Cardiothorac Surg 2016;50:1060-6. 
11. Delgado Roel M, Fieira Costa EM, González-Rivas D, et al. Uniportal video-assisted thoracoscopic lymph node dissection. J Thorac Dis 2014;6:S665-8.

12. Liu CC, Shih CS, Pennarun N, et al. Transition from a multiport technique to a single-port technique for lung cancer surgery: is lymph node dissection inferior using the single-port technique?. Eur J Cardiothorac Surg 2016;49 Suppl 1:i64-72.

13. Mu JW, Gao SG, Xue Q, et al. A Matched Comparison Study of Uniportal Versus Triportal Thoracoscopic Lobectomy and Sublobectomy for Early-stage Nonsmall Cell Lung Cancer. Chin Med J (Engl) 2015;128:2731-5.

14. Tamura M, Shimizu Y, Hashizume Y. Pain following thoracoscopic surgery: retrospective analysis between single-incision and three-port video-assisted thoracoscopic surgery. J Cardiothorac Surg 2013;8:153.

15. Lin F, Zhang C, Zhang Q, et al. Uniportal videoassisted thoracoscopic lobectomy: An alternative surgical method for pulmonary carcinoma. Pak J Med Sci 2016;32:1283-5.

16. Gonzalez D, de la Torre M, Paradela M, et al. Videoassisted thoracic surgery lobectomy: 3-year initial experience with 200 cases. Eur J Cardiothorac Surg 2011;40:e21-8.

17. Sihoe $\mathrm{AD}$. The evolution of minimally invasive thoracic surgery: implications for the practice of uniportal thoracoscopic surgery. J Thorac Dis 2014;6:S604-17.

18. Falcoz PE, Puyraveau M, Thomas PA, et al. Videoassisted thoracoscopic surgery versus open lobectomy for primary non-small-cell lung cancer: a propensitymatched analysis of outcome from the European Society of Thoracic Surgeon database. Eur J Cardiothorac Surg

Cite this article as: Pastina M, Menna C, Andreetti C, Ibrahim M. The era of uniportal video-assisted thoracoscopic surgery. J Thorac Dis 2017;9(3):462-465. doi: 10.21037/jtd.2017.02.97
2016;49:602-9.

19. Anile M, Diso D, Mantovani S, et al. Uniportal video assisted thoracoscopic lobectomy: going directly from open surgery to a single port approach. J Thorac Dis 2014;6:S641-3.

20. Stephens N, Rice D, Correa A, et al. Thoracoscopic lobectomy is associated with improved short-term and equivalent oncological outcomes compared with open lobectomy for clinical Stage I non-small-cell lung cancer: a propensity-matched analysis of 963 cases. Eur J Cardiothorac Surg 2014;46:607-13.

21. Harris CG, James RS, Tian DH, et al. Systematic review and meta-analysis of uniportal versus multiportal videoassisted thoracoscopic lobectomy for lung cancer. Ann Cardiothorac Surg 2016;5:76-84.

22. Dai F, Meng S, Mei L, et al. Single-port video-assisted thoracic surgery in the treatment of non-small cell lung cancer: a propensity-matched comparative analysis. J Thorac Dis 2016;8:2872-8.

23. French DG, Thompson C, Gilbert S. Transition from multiple port to single port video-assisted thoracoscopic anatomic pulmonary resection: early experience and comparison of perioperative outcomes. Ann Cardiothorac Surg 2016;5:92-9.

24. Chung JH, Choi YS, Cho JH, et al. Uniportal videoassisted thoracoscopic lobectomy: an alternative to conventional thoracoscopic lobectomy in lung cancer surgery? Interact Cardiovasc Thorac Surg 2015;20:813-9.

25. Gonzalez-Rivas D, Stupnik T, Fernandez R, et al. Intraoperative bleeding control by uniportal videoassisted thoracoscopic surgery†. Eur J Cardiothorac Surg 2016;49 Suppl 1:117-24. 